\title{
Glutamine-Loaded Liposomes: Preliminary Investigation, Characterization, and Evaluation of Neutrophil Viability
}

\author{
Larissa Chaves Costa, ${ }^{1,4}$ Bárbara Nayane Rosário Fernandes Souza, ${ }^{1}$ Fábio Fidélis Almeida, ${ }^{1}$ \\ Cláudia Jacques Lagranha, ${ }^{2}$ Pabyton Gonçalves Cadena, ${ }^{1,3}$ \\ Nereide Stela Santos-Magalhães, ${ }^{1,4}$ and Mariane Cajubá de Britto Lira-Nogueira ${ }^{1,2,5}$
}

Received 27 March 2015; accepted 16 July 2015; published online 31 July 2015

\begin{abstract}
Glutamine has received attention due to its ability to ameliorate the immune system response. Once conventional liposomes are readily recognized and captured by immune system cells, the encapsulation of glutamine into those nanosystems could be an alternative to reduce glutamine dosage and target then to neutrophils. Our goals were to nanoencapsulate glutamine into conventional liposomes (Gln-L), develop an analytical high-performance liquid chromatography (HPLC) method for its quantification, and evaluate the viability of neutrophils treated with Gln-L. Liposomes were prepared using the thin-film hydration technique followed by sonication and characterized according to $\mathrm{pH}$, mean size, zeta potential, and drug encapsulation efficiency (EE\%). We also aimed to study the effect of liposomal constituent concentrations on liposomal characteristics. The viability of neutrophils was assessed using flow cytometry after intraperitoneal administration of free glutamine (Gln), Gln-L, unloaded-liposome (UL), and saline solution as control (C) in healthy Wistar rats. The selected liposomal formulation had a mean vesicle size of $114.65 \pm 1.82 \mathrm{~nm}$ with a polydispersity index of $0.30 \pm 0.00$, a positive surface charge of $36.30 \pm 1.38 \mathrm{mV}$, and an $\mathrm{EE} \%$ of $39.49 \pm 0.74 \%$. The developed chromatographic method was efficient for the quantification of encapsulated glutamine, with a retention time at $3.8 \mathrm{~min}$. A greater viability was observed in the group treated with glutamine encapsulated compared to the control group (17\%), although neutrophils remain viable in all groups. Thus, glutamine encapsulated into liposomes was able to increase the number of viable neutrophils at low doses, thereby representing a promising strategy for the treatment of immunodeficiency conditions.
\end{abstract}

KEY WORDS: cell viability; glutamine; liposomes; neutrophils.

\section{INTRODUCTION}

Glutamine (Gln) is the most abundant free amino acid in plasma and muscle tissue and can also be found at relatively high concentrations in other tissues (1). The synthesis of this amino acid occurs primarily in skeletal muscle, as well as in the lung, liver, and brain. However, its consumption occurs primarily in the kidney, immune system, and gastrointestinal tract cells. The liver is the only organ able to consume and synthesize glutamine (2). Despite being classified as nonessential, glutamine is currently considered to be conditionally essential; in specific critical contexts, such as surgery, trauma,

\footnotetext{
${ }^{1}$ Laboratório de Imunopatologia Keizo-Asami, Universidade Federal de Pernambuco, Recife, PE, Brazil.

${ }^{2}$ Centro Acadêmico de Vitória, Universidade Federal de Pernambuco, Rua do Alto do Reservatório s/n, Bela Vista, CEP: 55608-680, Vitória de Santo Antão, PE, Brazil.

${ }^{3}$ Departamento de Morfologia e Fisiologia Animal, Universidade Federal Rural de Pernambuco, Recife, PE, Brazil.

${ }^{4}$ Departamento de Ciências Farmacêuticas, Universidade Federal de Pernambuco, Recife, PE, Brazil.

${ }^{5}$ To whom correspondence should be addressed. (e-mail: marianelira@gmail.com)
}

and exhaustive exercise, the synthesis of glutamine does not supply the demand required by the body, resulting in immunocompetence and an increased incidence of infections (3-5).

Glutamine plays a major role in cell proliferation particularly in the immune system, in which it has been used at high rates by lymphocytes as a source of energy, providing an ideal condition for the biosynthesis of nucleotides and thus cell replication (6). Regarding leukocytes, they are largely dependent on skeletal muscle glutamine synthesis and are also released into the blood to satisfy their metabolic requirements. This is due to the absence of glutamine synthetase, which catalyzes the synthesis of glutamine from ammonia and glutamate (7).

Neutrophils constitute $60 \%$ of circulating leukocytes and act as the first line of defense in the plasma and perform phagocytosis either alone or in cooperation with antigenspecific defenses. Processes of endocytosis, secretion of active compounds, and generation of reactive oxygen species are mostly dependent on glutamine metabolism in neutrophils (8).

Because neutrophils use glutamine at high rates compared to other cells of the immune system, researchers have investigated the effect of this amino acid on the cell parameters of human and rat neutrophils $(9,10)$. 
Moreover, glutamine is a component of proteins, it transports ammonia between tissues, is a source of carbon skeleton for gluconeogenesis, contributes to acid-base balance, and participates in the synthesis of nucleotides and nucleic acids $(4,11)$; thus, it is of great importance for the maintenance and proper functioning of an organism.

The events of metabolic stress or diseases, such as dengue, cancer, AIDS, burns, surgeries, and intense and prolonged exercise, are some situations in which there is an excessive consumption of glutamine, surpassing its synthesis by the body (12). Previous studies have demonstrated that a decrease in the intramuscular and plasma concentrations of glutamine may be partially responsible for the depression of immunological function and the administration of glutamine may reduce this state (13-15), thereby providing an improved recovery of patients with such diseases $(16,17)$.

Despite presenting significant benefits in some situations, previous studies have reported that glutamine supplementation can promote renal failure and may exacerbate the clinical situation of chronic kidney disease, particularly in diabetic patients $(11,18,19)$. Moreover, it has a solubility in water of $48 \mathrm{~g} / \mathrm{l}$ at $30^{\circ} \mathrm{C}\left(\mathrm{pKa}_{1}=2.17\right.$ and $\left.\mathrm{pKa}_{2}=9.13\right)$ and is unstable in solution, which may undergo breakdown depending on temperature, $\mathrm{pH}$, and anion concentration $(20,21)$. An approach to overcome these physicochemical limitations and to reduce adverse effects, while increasing its therapeutic effect, is the development of a pharmaceutical dosage form that is able to target the bioactive compound to a specific site of action, thereby increasing therapeutic efficacy. Thus, drug delivery systems, such as liposomes, may offer advantages to overcome these limitations.

Liposomes are spherical vesicles consisting of one or more concentric bilayers of phospholipids that isolate one or more aqueous inner compartments of the external environment (22). Liposomes are classified as conventional and consist of phospholipids and cholesterol; this type is quickly recognized and taken up by the immune system and is ideal for carrying drugs to neutrophils (23). We propose that the use of glutamine-loaded conventional liposomes could be a promising approach to target this amino acid to neutrophils, thereby enhancing their activity.

Within this framework, the goal of this research was to first develop conventional liposomes containing glutamine and to select a typical formulation after experimental design. Second, we aimed to develop an analytical method to determine and quantify the nanoencapsulated glutamine using high-performance liquid chromatography (HPLC), and finally, we evaluated the neutrophil viability, after the administration of glutamine-loaded conventional liposomes in Wistar rats.

\section{MATERIAL AND METHODS}

\section{Reagents}

L-Glutamine (Gln $\geq 99 \%)$, cholesterol (Chol), stearylamine (SA), glycogen from oyster type II, and propidium iodide were purchased from Sigma-Aldrich Co. (St. Louis, MO, USA). 98\% Soya phosphatidylcholine (PC) was obtained from Lipoid GmbH (Ludwigshafen, Germany).
Solvents and other chemicals were supplied by Merck (Darmstadt, Germany).

\section{Methodology \\ Experimental Design}

First, a two-level $2^{4-1}$ fractional experimental design was performed to study the effect of constituent concentrations on the properties of liposomes. Four formulation factors, which mainly affect liposome physicochemical characteristics, were evaluated at two levels and a central point. The factors were defined as follows: A $[\mathrm{PC}]=15.63,31.27$, and $46.91 \mathrm{mM}$; B $[\mathrm{Chol}]=4.42,8.84$, and $13.26 \mathrm{mM} ; \mathrm{C}[\mathrm{SA}]=0,4.19$, and $8.38 \mathrm{mM}$; and D [Gln] $=27.37,41.05$, and $54.74 \mathrm{mM}$.

The $\mathrm{pH}$, mean size of the vesicles, polydispersity index (PDI), zeta potential, $(\zeta, \mathrm{mV})$, and drug encapsulation efficiency $(\mathrm{EE} \%)$ of the liposomes were analyzed, as described below, and used as the response variables of the design study.

Next, a two-level $2^{2}$ full experimental design was performed to study the effect of PC and Chol concentrations, as well as their second-order interactions, on the physicochemical characteristics. The design was performed using a matrix of seven experiments with a central point, and the factors defined were as follows: $\mathrm{A}[\mathrm{PC}]=31.27,39.09$, and $46.91 \mathrm{mM}$ and $\mathrm{B}[\mathrm{Chol}]=8.84,11.05$, and $13.26 \mathrm{mM}$.

The same response variables of the fractional design study were used to analyze the full experimental design study.

\section{Preparation of Glutamine-Loaded Conventional Liposomes}

Glutamine-loaded liposomes were prepared using the modified thin-lipid-film method (24). Briefly, lipids consisting of soya phosphatidylcholine and cholesterol, with or without stearylamine, a positive charged lipid, were dissolved in a mixture of $\mathrm{CHCl}_{3}: \mathrm{MeOH}(3: 1 \mathrm{v} / \mathrm{v})$ under magnetic stirring. The solvents were removed under pressure at $37 \pm 1^{\circ} \mathrm{C}, 80 \mathrm{rpm}$ for $60 \mathrm{~min}$, forming a thin lipid film. This film was then hydrated with $10 \mathrm{ml}$ of phosphate buffer solution at $\mathrm{pH} 7.4$, containing glutamine $(27.37,41.05$, and $54.74 \mathrm{mM})$ that had been previously dissolved, resulting in the formation of multilamellar liposomes. Finally, the liposomal suspension was sonicated (Vibra Cell, Branson, USA) at $200 \mathrm{~W}$ and $40 \mathrm{~Hz}$ for $400 \mathrm{~s}$ under low temperature $\left(4^{\circ} \mathrm{C}\right)$ to form small unilamellar liposomes.

\section{Physicochemical Characterization of Glutamine-Loaded Con- ventional Liposomes}

Liposome formulations were characterized by the measurement of $\mathrm{pH}$, mean hydrodynamic diameter, PDI, and surface charge (zeta potential) of vesicles. The $\mathrm{pH}$ of the liposome dispersions was measured using a digital $\mathrm{pH}$ meter (Micronal B474, São Paulo, SP, Brazil) at $25^{\circ} \mathrm{C}$. The polydispersity index and diameter of the liposomes were quantified using photon correlation spectroscopy (Delsa ${ }^{\mathrm{TM}}$ Nano S Particle analyzer, Beckman Coulter, Brea, CA, USA), at $25^{\circ} \mathrm{C}$ at a fixed angle of $90^{\circ}$. For the analysis, the samples were adequately diluted using $300 \mu \mathrm{l}$ of liposomes and $700 \mu \mathrm{l}$ of purified water. The zeta potential $(\zeta, \mathrm{mV})$ was measured at $25^{\circ} \mathrm{C}$ using the electrophoresis technique (Malvern Zetasizer Nano ZS90, 
Malvern Instruments Ltd., Worcestershire, UK). In these analyses, liposome dispersions $(50 \mu \mathrm{l})$ were diluted with $950 \mu \mathrm{l}$ of purified water. The results represent the average of three determinations.

The drug EE\% was determined using the ultrafiltration/ ultracentrifugation technique with Ultrafree ${ }^{\circledR}$ units (Millipore Corporation, Bedford, MA, USA) (24). Liposomal samples $(400 \mu \mathrm{l})$ were inserted into the filtration unit and subjected to centrifugation (Ultracentrifuge KT-20000, Kubota, Japan) at $8776 \times g$ for $1 \mathrm{~h}$ at $4^{\circ} \mathrm{C}$. The content of Gln in the supernatant was measured using the HPLC method as detailed below.

\section{Extraction and Determination of the Glutamine Content into Conventional Liposomes}

The method for glutamine extraction was developed according to the two-phase system previously described by Bligh and Dyer (25), which obtained the organic (lipids) and aqueous (glutamine) phases. Briefly, for Gln extraction, $200 \mu \mathrm{l}$ of liposomes was diluted into $400 \mu$ l of a mixture of $\mathrm{CHCl}_{3}: \mathrm{MeOH}(1: 1)$ and subsequently sonicated for $3 \mathrm{~min}$. Next, 1 and $1.7 \mathrm{ml}$ of chloroform and methanol, respectively, were added and sonicated for more than $3 \mathrm{~min}$. For the twophase system formation, purified water was added to a final volume of $5 \mathrm{ml}$. After visual separation, the aqueous fraction was filtered and analyzed, to determine the glutamine content, using the HPLC method.

The HPLC method for determining and quantifying the glutamine content into liposomes was performed using a liquid chromatographer (Waters Alliance e2695-2998, Waters, Milford, MA, USA) coupled with a photodiode array detector and operated by Empower ${ }^{\mathrm{TM}}$ software. The chromatographic run was performed using a reversed-phase Waters C18 $\mathrm{XBridge}^{\circledR}$ column $(4.6 \times 250 \mathrm{~mm}$ i.d. particle $3.5 \mu \mathrm{m})$ with $50 \mu \mathrm{l}$ of sample injection volume at $37^{\circ} \mathrm{C}$. HPLC analysis of Gln was performed using a mobile phase consisting of methanol and double-deionized water $(6: 4 v / v)$ at a flow rate of $0.7 \mathrm{ml} / \mathrm{min}$ and detection at $204 \mathrm{~nm}$. Standard curves were assayed using glutamine solutions at concentrations ranging from 80 to $400 \mu \mathrm{g} / \mathrm{ml}$. The assays were performed in triplicate.

\section{In Vivo Experiment}

\section{Animals}

The in vivo study was performed according to Lagranha and co-workers (10). This study was performed according to the Ethics Committee for Experiments on Animals of the Federal University of Pernambuco (CEUA-UFPE, Recife, Brazil) with protocol approval (\#23076.013231/2012-47). Female Wistar rats $(200 \pm 20 \mathrm{~g})$ were maintained in collective cages until the day of the experiment, at a temperature of 22 $\pm 2^{\circ} \mathrm{C}$ and relative humidity of $60 \%$ and under a cycle of $12 \mathrm{~h}$ light/12 h darkness with unrestricted access to water and food (commercial chow). The animals were randomly divided into five following groups of three rats each: Gln-IP (glutamine in solution by intraperitoneal route), Gln-GAV (glutamine in solution by gavage), Gln-L (glutamine-loaded liposomes), UL (unloaded liposomes), and C (control/saline as placebo). The rats did not present a metabolic disorder, such as diabetes, and were not subjected to intense exercise or surgical procedures.

\section{Recruitment of Neutrophils}

With the purpose of causing an inflammatory stimulus and the substantial migration of neutrophils into the intraperitoneal cavity, $15 \mathrm{ml}$ of sterile oyster glycogen solution type II (1\%) in PBS was administered intraperitoneally to the rats prior to the experiments.

\section{Treatment}

After $2 \mathrm{~h}$ of neutrophil recruitment, the rats received free Gln, Gln-L, UL, and saline solution as the C. Glutamine in its free form was administered via two different routes: orally (1 g/kg) according to Lagranha and colleagues $(10,26)$ and intraperitoneally $(0.06 \mathrm{~g} / \mathrm{kg})$. Gln-L $(0.06 \mathrm{~g} / \mathrm{kg})$, UL, and saline solution were administered intraperitoneally $(2 \mathrm{ml}$ each, with the same volume, which corresponded to the glutamineloaded liposomes).

\section{Collecting Neutrophils}

Rats were anesthetized with Urethane ${ }^{\circledR}(1.25 \mathrm{~g} / \mathrm{kg})$ and sacrificed by cervical dislocation $2 \mathrm{~h}$ after treatment. Neutrophils were obtained by intraperitoneal (i.p.) lavage with $40 \mathrm{ml}$ sterile phosphate-buffered saline. The cells were centrifuged (1000×g for $10 \mathrm{~min}$ ), washed twice with PBS, and then quantified in a Neubauer chamber using the Trypan Blue solution (1\% in PBS) and optical microscopy (Olympus, USA).

\section{Neutrophil Viability Assay}

The viability of neutrophils was assessed using a flow cytometer (FACSCalibur, Becton Dickinson Systems, San Jose, CA, USA). The percentage of viable cells in each sample was determined using propidium iodide staining (solution at $0.05 \%$ in PBS) to identify dead cells. Data of 15.000 events were analyzed per sample. The fluorescence of the propidium iodide was detected at 630/622 $\mathrm{nm}$.

\section{Statistical Analysis}

Experimental designs were performed in a random order. Statistical analyses were achieved using the OriginPro Academic 2015 (Origin Lab., Northampton, MA, USA). Statistically significant differences were determined using the Tukey multiple comparisons test, with $p$ values less than 0.05 as the minimal level of significance.

\section{RESULTS AND DISCUSSION}

\section{Experimental Design of Liposomal Formulations}

Several factors related to the development of nanosystems may affect their characteristics, and a small change in even one factor may significantly change the properties of the entire system. According to the strategy of Cadena and colleagues (27), two experimental designs were performed aimed to study the effect of four factors in the formulation, i.e., the 
concentration of constituents on the physicochemical properties of Gln-L, thereby obtaining optimized formulations.

The experiments were randomly assayed to nullify the effect of inappropriate nuisance variables in both experimental designs. The nine runs of the two-level $2^{4-1}$ fractional experimental design and their response variables are shown in Table I. Each response variable was individually analyzed, and it was observed that a lower level of stearylamine (SA) and a higher level of soya phosphatidylcholine (PC) reduced the mean size of the vesicles (runs 2 and 4). However, a higher level of SA increased the $\mathrm{pH}$ (runs 5 and 8 ) and a higher level of SA, PC, and Chol reduced the PDI values (run 8). It was also observed that a higher level of SA increased the zeta potential (runs 5 and 8 ). The glutamine encapsulation efficiency $(\mathrm{EE} \%)$ was not affected by the factors studied $(p<0.05)$. The higher Gln level (54.74 $\mathrm{mM}$ ) and central point $(41.05 \mathrm{mM})$ presented no statistically significant difference on the EE\% of $43.20 \pm 0.86$ and $38.74 \pm 0.78 \%$, respectively. However, run 8 had a mean size of $315.71 \pm 16.2$, which is higher than the run found in the central point (177.7 $\pm 7.8 \mathrm{~nm}$ ). Thus, a concentration of $41.05 \mathrm{mM}$ Gln was used in further experiments due to the smaller size of liposomes.

A scatterplot (Fig. 1) was used to identify the best liposomal formulation obtained in the fractional experimental design study, in which the central point (run 9) showed a zeta potential of $32.73 \pm 0.63 \mathrm{mV}$, an $\mathrm{EE} \%$ of $38.74 \pm 0.78$, and a mean vesicle size of $177.7 \pm 7.76$, and maintained its stability in suspension for at least 45 days. Finally, run 9 was used as the typical formulation for the new experimental design.

The two-level $2^{2}$ full experimental design was developed to study the effect of PC and Chol concentrations and their interactions on liposomal characteristics, once they are the main relevant lipids, which directly affected the physicochemical parameters studied above.

The concentration of SA $(4.19 \mathrm{mM})$ was maintained because it did not statistically demonstrate significant differences $(p<0.05)$ between the higher level and central point in the zeta potential, reducing the amount of SA used. Secondly, the presence of SA promoted a zeta potential value above

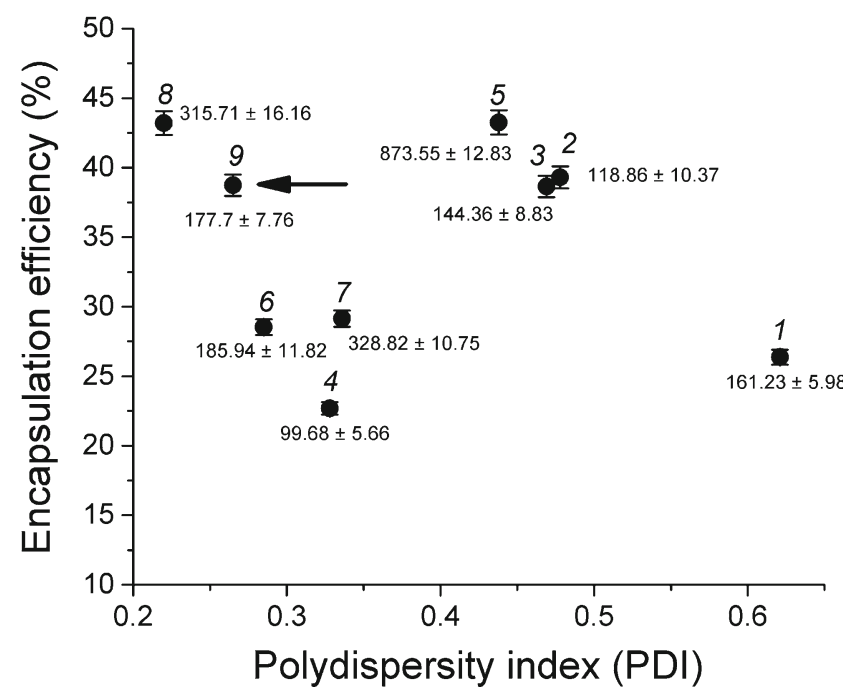

Fig. 1. Scatterplot of the formulations produced using a $2^{4-1}$ fractional experimental design and the effects of response variables: polydispersity index (PDI) and drug encapsulation efficiency (\%). The arrow points the best liposomal formulation. Formulation run numbers and the mean size $(\mathrm{nm})$ of liposomes are indicated above each point

$30 \mathrm{mV}$ (runs 5-9), which, as reported in the literature, is an ideal value (even negative or positive) to avoid vesicle aggregation by electrostatic repulsion (28).

Table I shows the results obtained in the design study. It was observed that only the mean size was significantly affected by PC and Chol concentrations $(p<0.05)$, where the PC had the main statistically significant negative effect, and the higher level of PC (46.91 mM) decreased the mean size of the liposomes. The second-order interactions between the PC and $\mathrm{CH}$ concentration values had a positive effect, meaning that the main mean size was slightly enhanced by raising the levels of the two factors together. The results are shown in Table II.

With regard to the hydrophilic drug EE\%, previous studies have reported values at approximately 14 to $23 \%$ as described by Wieber and colleagues (29), when encapsulating a

Table I. Experimental Design and Physicochemical Properties of Glutamine-Loaded Liposomes

\begin{tabular}{|c|c|c|c|c|c|c|c|c|c|}
\hline Runs & $\mathrm{PC}(\mathrm{mM})$ & Chol (mM) & $\mathrm{SA}(\mathrm{mM})$ & $\mathrm{Gln}(\mathrm{mM})$ & $\mathrm{pH}$ & Size $(\mathrm{nm})$ & PDI & $\zeta(\mathrm{mV})$ & $\mathrm{EE}(\%)$ \\
\hline \multicolumn{10}{|c|}{ A. Two-level $2^{4-1}$ fractional experimental design } \\
\hline 1 & 15.63 & 4.42 & 0 & 27.37 & 7.49 & $161.23 \pm 5.98$ & $0.621 \pm 0.00$ & $0.01 \pm 0.07$ & $26.37 \pm 0.53$ \\
\hline 2 & 46.91 & 4.42 & 0 & 54.74 & 7.43 & $118.86 \pm 10.37$ & $0.478 \pm 0.03$ & $-14.73 \pm 1.19$ & $39.30 \pm 0.79$ \\
\hline 3 & 15.63 & 13.26 & 0 & 54.74 & 7.49 & $144.36 \pm 8.83$ & $0.469 \pm 0.04$ & $1.94 \pm 0.14$ & $38.66 \pm 0.77$ \\
\hline 4 & 46.91 & 13.26 & 0 & 27.37 & 7.54 & $99.68 \pm 5.66$ & $0.328 \pm 0.01$ & $5.73 \pm 0.30$ & $22.68 \pm 0.45$ \\
\hline 5 & 15.63 & 4.42 & 8.38 & 54.74 & 7.58 & $873.55 \pm 12.83$ & $0.438 \pm 0.01$ & $31.96 \pm 1.35$ & $43.25 \pm 0.87$ \\
\hline 6 & 46.91 & 4.42 & 8.38 & 27.37 & 7.62 & $185.94 \pm 11.82$ & $0.285 \pm 0.02$ & $33.73 \pm 1.58$ & $28.53 \pm 0.57$ \\
\hline 7 & 15.63 & 13.26 & 8.38 & 27.37 & 7.65 & $328.82 \pm 10.75$ & $0.336 \pm 0.01$ & $31.40 \pm 1.21$ & $29.14 \pm 0.58$ \\
\hline 8 & 46.91 & 13.26 & 8.38 & 54.74 & 7.68 & $315.71 \pm 16.16$ & $0.220 \pm 0.01$ & $33.20 \pm 1.25$ & $43.20 \pm 0.86$ \\
\hline 9 (cp) & 31.27 & 8.84 & 4.19 & 41.05 & 7.54 & $177.7 \pm 7.76$ & $0.265 \pm 0.01$ & $32.73 \pm 0.63$ & $38.74 \pm 0.78$ \\
\hline \multicolumn{10}{|c|}{ B. Two-level $2^{2}$ full experimental design } \\
\hline 1 & 31.27 & 8.84 & 4.19 & 41.05 & 7.74 & $165.66 \pm 3.09$ & $0.297 \pm 0.01$ & $39.50 \pm 1.91$ & $44.67 \pm 0.26$ \\
\hline 2 & 31.27 & 13.26 & 4.19 & 41.05 & 7.76 & $130.36 \pm 3.42$ & $0.307 \pm 0.01$ & $36.30 \pm 0.10$ & $32.91 \pm 0.34$ \\
\hline 3 & 46.91 & 8.84 & 4.19 & 41.05 & 7.75 & $85.51 \pm 2.80$ & $0.294 \pm 0.00$ & $32.93 \pm 1.01$ & $7.35 \pm 1.00$ \\
\hline 4 & 46.91 & 13.26 & 4.19 & 41.05 & 7.75 & $96.16 \pm 1.23$ & $0.274 \pm 0.01$ & $36.90 \pm 1.50$ & $28.29 \pm 0.07$ \\
\hline $5(\mathrm{cp})$ & 39.09 & 11.05 & 4.19 & 41.05 & 7.69 & $114.65 \pm 1.82$ & $0.300 \pm 0.00$ & $36.30 \pm 1.38$ & $39.49 \pm 0.74$ \\
\hline
\end{tabular}

$P C$ phosphatidylcholine, Chol cholesterol, $S A$ stearylamine, Gln glutamine, PDI polydispersity index, $\zeta$ surface charge, EE encapsulation efficiency 
Table II. ANOVA Table for the Full Experimental Design

\begin{tabular}{|c|c|c|c|c|c|}
\hline Factors & Sums of square & Degrees of freedom & Mean square & $F$ & $p$ value \\
\hline (1) $\mathrm{PC}(\mathrm{mM})^{a}$ & 3268.98 & 1 & 3268.98 & 122.87 & 0.008041 \\
\hline (2) Chol (mM) & 151.90 & 1 & 151.90 & 5.71 & 0.139429 \\
\hline 1 by $2^{a}$ & 527.85 & 1 & 527.85 & 19.84 & 0.046887 \\
\hline Error & 133.13 & 1 & 133.13 & 5.00 & 0.154750 \\
\hline Total & 53.21 & 2 & 26.60 & & \\
\hline
\end{tabular}

PC phosphatidylcholine, Chol cholesterol

${ }^{a}$ Significant response

decapeptide with a concentration varying from 0.25 to $0.75 \mathrm{mg} / \mathrm{ml}$. Xu and colleagues (2012) also demonstrated a low encapsulation efficiency for hydrophilic compounds, such as the drug tenofovir ( $\mathrm{Mw} 287.2113$ ). The authors varied the lipid concentrations and observed that lowering the amount of lipids promoted low drug encapsulation, while very high lipid concentrations (152.9 mM) showed an EE\% of 9 to $45 \%$ using a drug concentration of $0.5,1,2.5$, and $6.9 \mathrm{mg} / \mathrm{ml}$. Importantly, a high amount of some lipids, particularly stearylamine, in liposome formulations could be toxic for cells (30). Our results corroborate with previous work in this field, in which we also obtained low encapsulation efficiency. However, in contrast to other studies that used a low drug concentration, we varied the glutamine concentration from 4 to $8 \mathrm{mg} / \mathrm{ml}$, thereby obtaining a maximum encapsulation efficiency of $44 \%$, which is a significant result.

On the basis of the results obtained in the two-level $2^{2}$ full experimental design, specifically related to $\mathrm{EE} \%$, the central point (run 5 and $\mathrm{EE} \%$ of $39.49 \pm 0.74 \%$ ) was selected for the in vivo experiments, which had the lowest size (114.65 $\pm 1.82 \mathrm{~nm})$ and an acceptable PDI $(0.300 \pm 0.00)$. After 45 days, in suspension form under low temperature $\left(4^{\circ} \mathrm{C}\right)$, the formulation presented a mean size of $180 \pm 4.90 \mathrm{~nm}$ (PDI $=0.30 \pm$ $0.01)$.

\section{Determination of Glutamine Content into Liposomes}

In our study, as the glutamine was encapsulated into liposomes, which contained lipids, it was necessary to develop an efficient extraction method in parallel to an HPLC method, which would be able to quantify the glutamine without lipid interference, at a wavelength ranging from 190 to $210 \mathrm{~nm}$ (31). Thus, an extraction method based on the two-phase liquidliquid system was first obtained to separate the aqueous and organic phases of the liposomes, which contained glutamine and lipids, respectively. Samples from the aqueous phase were withdrawn and analyzed using HPLC according to the parameters previously described. The chromatograms of glutamine, glutamine-loaded liposomes, and unloaded liposomes are shown in Fig. 2a, b. The standard curve of glutamine was plotted with nine concentrations and Gln peak areas allowing a following regression linear equation: $A=16348.56 \times\left[\mathrm{G} \ln _{\mu \mathrm{g}}\right.$ / $\mathrm{ml}]-19853.12 ; r^{2}=0.99514$.

As observed, free glutamine had a retention time of approximately $3.80 \mathrm{~min}$, and the same retention time was observed in the glutamine-loaded liposome chromatogram. Compared to the unloaded liposomes, no peak was observed at this retention time $(3.8 \mathrm{~min})$, and any peak related to lipids suggested an adequate separation of glutamine, which enabled its quantification of liposome formulations.

Amino acid analysis by HPLC presents limitations that are specifically related to sensitive and selective detection, in addition to a wide range of wavelengths varying from 205 to $230 \mathrm{~nm}$ (32). For this reason, studies related to the development of an adequate HPLC method, aimed to analyze glutamine in different systems, were widely found in the literature. Snowden and
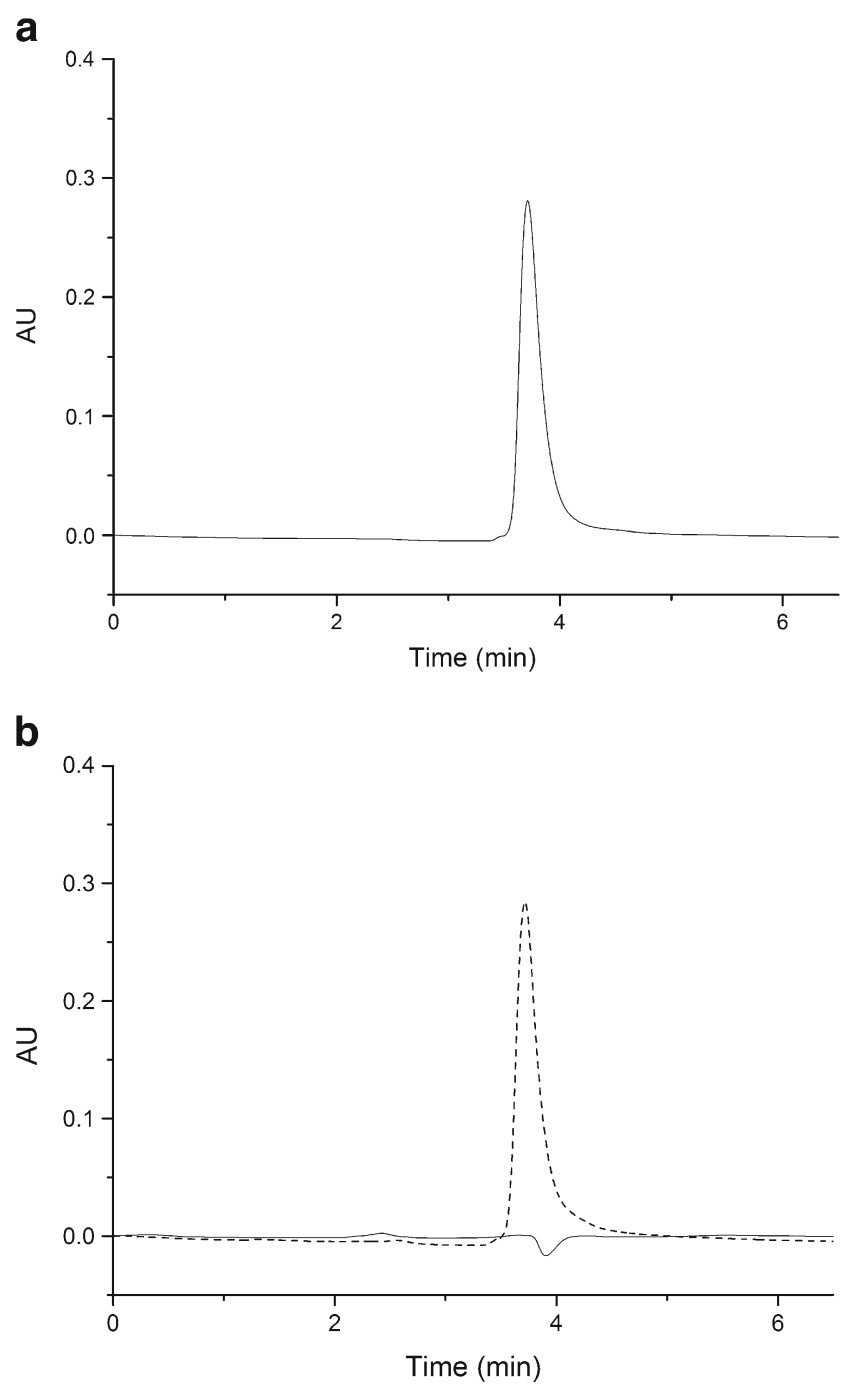

Fig. 2. Chromatographic detection of free and nanoencapsulated glutamine. Glutamine in a solvent mixture of $\mathrm{H}_{2} \mathrm{O}: \mathrm{MeOH}$ with a retention time of $3.8 \mathrm{~min}$ (a). Comparison between glutamine-loaded liposomes (broken line) and unloaded liposomes (solid line) (b) 
co-workers (33) described a HPLC method using $214 \mathrm{~nm}$ as an ideal wavelength to quantify glutamine and $N$-acetylglutamine in an aqueous solution and liquid nutritional products. Another method was the one recently described by Chorilli et al. (34). They published a different method to analyze amino acids using ion exchange chromatography followed by post-column derivatization. Using this method, they were able to detect glutamine outside the usual wavelength, at $530 \mathrm{~nm}$ and at a retention time of $10 \mathrm{~min}$. The extraction and quantification methods were developed, as previously described, once the methods described in the literature were not able to quantify glutamine in the liposomal form.

Thus, the extraction method was efficient and the HPLC developed method was used to determine the glutamine encapsulated into liposomes consisting of soya phosphatidylcholine, cholesterol, and stearylamine.

\section{Neutrophil Viability Assay}

Several studies have been performed to indicate the effectiveness of glutamine in increasing the immunity of rats immunosuppressed by intense and prolonged exercise. In studies developed by Lagranha et al. $(26,35)$, they demonstrated that a single bout of exercise induced the apoptosis of rat neutrophils and oral glutamine supplementation $(1 \mathrm{~g} / \mathrm{kg})$ prevented this effect, by promoting an increase in neutrophil phagocytosis and oxidative capacity from both exercised and rested rats.

Despite the efficacy related to neutrophil activation, glutamine at high concentrations can promote nephrotoxicity, particularly in diabetic patients $(18,19)$. Thus, the use of glutamine encapsulated into nanostructured delivery systems, such as conventional liposomes, could decrease the dose as well as the toxicity, once conventional liposomes are able to target the encapsulated compound directly to neutrophils. This study describes for the first time the analysis of neutrophil viability in rats after the administration of glutamine-loaded conventional liposomes. Our results showed that all rat groups investigated presented a high percentage of intact plasma membrane as observed in Fig. 3, once the animals were considered healthy, which means they did not present a metabolic disorder, such as diabetes, and were not subjected to intense exercise or surgical procedures.

Regarding unloaded liposomes, a high rate of neutrophil viability $(87 \pm 2.7 \%)$ was observed compared to the control group. Because liposomes are formed by phospholipid bilayers, it has been reported that these vesicles are able to mimic the shape and size of pathogenic microorganisms (36) and consequently promote an increase in the immune response of animals (37). Thus, unloaded liposomes may be recognized as a foreign body and stimulate the immune response, explaining the increased value of neutrophils in the treated animals.

Peritoneal administration of glutamine-loaded conventional liposomes (Gln-L) and unloaded liposomes presented a viability of $94 \pm 0.2$ and $87 \pm 2.7 \%$, respectively. Those data demonstrate that Gln-L promoted an increase in neutrophil viability of 21 and $8.2 \%$ compared to the control and unloaded-liposomes groups (Fig. 3).

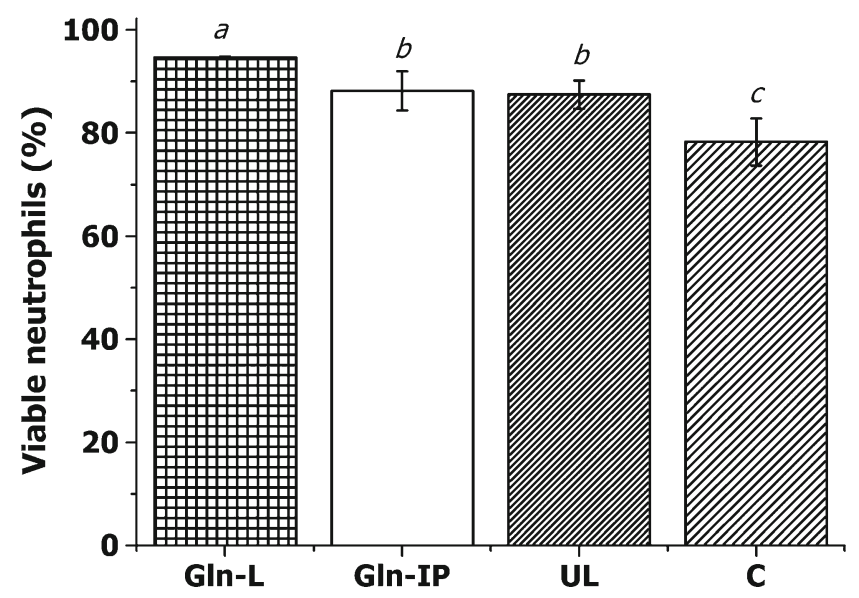

Fig. 3. Effect of glutamine on neutrophil membrane integrity. The percentage of viable neutrophils in response to different treatments via the intraperitoneal route. These values are presented as the mean $\pm \mathrm{SD}$ of three experiments $(n=3)$. Average data with different letters are significantly different using the Tukey test $(p<0.05)$. Gln- $L$ glutamineloaded liposomes, Gln glutamine in solution, $U L$ unloaded liposomes, $C$ control group

Interestingly, when comparing the Gln-L with the Gln group, an increase in cell viability $(7.3 \%)$ was observed, demonstrating a positive effect when glutamine was encapsulated into liposomes. This remarkable finding was related to nonviable neutrophils, once the Gln-L group presented the lowest percentage $(5 \pm 0.5 \%)$, thereby confirming that this system did not cause cellular damage.

A comparison between free Gln administered using two different routes, oral and intraperitoneal, showed no statistical differences in the percentage of viable cells $(87 \pm 6.4$ and 88 $\pm 3.8 \%$, respectively) and nonviable cells $(9 \pm 1.2$ and $5 \pm 1.4 \%$, respectively) (Fig. 4). This finding indicates that a reduced dosage of 17-fold via the intraperitoneal route achieved the same effect as a higher dosage via oral supplementation $(10,14,35)$.

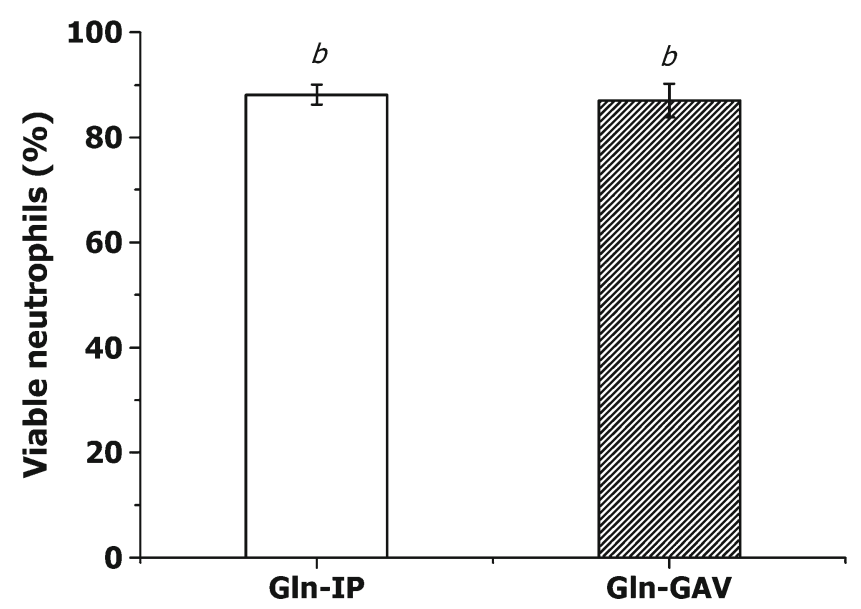

Fig. 4. Comparison between free glutamine in solution administered via the oral $(G \ln -G A V)$ and intraperitoneal $(G l n-I P)$ routes. The values are presented as the mean $\pm \mathrm{SD}$ of three experiments $(n=3)$. Average data with different letters are significantly different using the Tukey test $(p<0.05)$ 


\section{CONCLUSIONS}

Glutamine-loaded liposomes were obtained and presented desirable physicochemical characteristics, such as $\mathrm{pH}$, mean size, polydispersity index, zeta potential, and drug encapsulation efficiency. The experimental factorial designs performed indicated the ideal concentration of the formulation constituents and ideal formulation.

This study also described for the first time a sufficient HPLC method for the quantification of glutamine encapsulated into liposomes. Glutamine-loaded liposomes administered via the i.p. route were able to increase neutrophil viability with very low nonviable cells in healthy animals. On the basis of these findings, this study encourages the use of formulations developed in further studies using immunosuppressed animals, which are aimed to increase immunological function.

\section{ACKNOWLEDGMENTS}

The authors thank the Brazilian National Council for Research (CNPq) for financial support (\#484574/2011-6) and the Foundation for Science and Technology of Pernambuco (FACEPE). Thanks are also due to the Keizo Asami Immunopathology Laboratory (LIKA).

\section{REFERENCES}

1. Newsholme P, Lima MMR, Procopio J, Pithon-Curi TC, Doi SQ, Bazotte RB, et al. Glutamine and glutamate as vital metabolites. Braz J Med Biol Res. 2003;36:153-63.

2. Labow BI, Souba WW. Glutamine. World J Surg. 2000;24:150313. doi:10.1007/s002680010269.

3. Albino Junior W, Alberto C, Taliari K, Cancelliero KM. Suplementação com glutamina melhora as reservas de glicogênio de músculos de ratos tratados com dexametasona. Rev Bras Educ Fís Esp. 2004;18:283-91.

4. Cruzat VF, Petry ER, Tirapegui J. Glutamina : aspectos bioquímicos, metabólicos, moleculares e suplementação. Rev Bras Med Esporte. 2009;15:392-7.

5. Harris RC, Hoffman JR, Allsopp A, Routledge NBH. Lglutamine absorption is enhanced after ingestion of Lalanylglutamine compared with the free amino acid or wheat protein. Nutr Res. 2012;32:272-7. doi:10.1016/ j.nutres.2012.02.003.

6. Gleeson M. Dosing and efficacy of glutamine supplementation in human exercise and sport training. J Nutr. 2008;138:2045S-9S.

7. Ardawi MSM, Newsholme EA. Glutamine metabolism in lymphocytes of the rat. Biochem J. 1983;212:835-42.

8. Curi TCP, Melo MP, Azevedo RB, Zorn TMT, Curi R. Glutamine utilization by rat neutrophils: presence of phosphatedependent glutaminase. Am J Physiol Cell Physiol. 1997;273:1124-9.

9. Pithon-Curi TC, Schumacher RI, Freitas JJS, Lagranha C, Newsholme P, Palanch AC, et al. Glutamine delays spontaneous apoptosis in neutrophils. Am J Physiol Cell Physiol. 2003;284:C1355-61. doi:10.1152/ajpcell.00224.2002.

10. Lagranha CJ, Senna SM, Lima TM, Silva EPP, Doi SQ, Curi R, et al. Beneficial effect of glutamine on exercise-induced apoptosis of rat neutrophils. Med Sci Sports Exerc. 2004;36:210-7. doi:10.1249/01.MSS.0000113490.98089.B1.

11. Curi R, Lagranha CJ, Doi SQ, Sellitti DF, Procopio J, Pithon-Curi TC, et al. Molecular mechanisms of glutamine action. J Cell Physiol. 2005;204:392-401. doi:10.1002/jcp.20339.

12. Lacey JM, Wilmore DW. Is glutamine a conditionally essential amino acid? Nutr Rev. 1990;48:297-309.

13. Furst P. New developments in glutamine delivery. J Nutr. 2001;131:2562-8.
14. Lagranha CJ, Levada-Pires AC, Sellitti DF, Procopio J, Curi R, Pithon-Curi TC. The effect of glutamine supplementation and physical exercise on neutrophil function. Amino Acids. 2008;34:337-46. doi:10.1007/s00726-007-0560-x.

15. Fan Y, Yu J, Kang W, Zhang Q. Effects of glutamine supplementation on patients undergoing abdominal surgery. Chin Med Sci J. 2009;24:55-9. doi:10.1016/S1001-9294(09)60060-2.

16. Wilmore DW. The effect of glutamine supplementation in patients following elective surgery and accidental injury. J Nutr. 2001;131:2543-9.

17. Fläring UB, Rooyackers $\mathrm{OE}$, Wernerman J, Hammarqvist F. Glutamine attenuates post-traumatic glutathione depletion in human muscle. Clin Sci (Lond). 2003;104:275-82. doi:10.1042/ CS20020198.

18. Alba-Loureiro TC, Ribeiro RF, Zorn TMT, Lagranha CJ. Effects of glutamine supplementation on kidney of diabetic rat. Amino Acids. 2010;38:1021-30. doi:10.1007/s00726-009-0310-3.

19. Galera SC, Fechine FV, Teixeira MJ, Coelho ZCB, Vasconcelos RC, Vasconcelos PRL. The safety of oral use of L-glutamine in middle-aged and elderly individuals. Nutrition. 2010;26:375-81. doi:10.1016/j.nut.2009.05.013.

20. D'Souza R, Powell-Tuck J. Glutamine supplements in the critically ill. J R Soc Med. 2004;97:425-7. doi:10.1258/jrsm.97.9.425.

21. O'Neil MJ. The Merck Index: an encyclopedia of chemicals, drugs, and biologicals. 6th ed. New Jersey: Merck; 2006.

22. Brandl M, Gregoriadis G. Entrapment of haemoglobin into liposomes by the behaviour. Biochim Biophys Acta. 1994;1196:65-75.

23. Torchilin VP. Recent advances with liposomes as pharmaceutical carriers. Nat Rev Drug Discov. 2005;4:145-60. doi:10.1038/ $\operatorname{nrd} 1632$.

24. Lira MCB, Ferraz MS, Silva DGVC, Cortes ME, Teixeira KI, Caetano NP, et al. Inclusion complex of usnic acid with $\beta$-cyclodextrin: characterization and nanoencapsulation into liposomes. J Incl Phenom Macrocycl Chem. 2009;64:215-24. doi:10.1007/ s10847-009-9554-5.

25. Bligh EG, Dyer WJ. A rapid method of total lipid extraction and purification. Can J Biochem Physiol. 1959;37:911-7.

26. Lagranha CJ, Alba-Loureiro TC, Martins EF, Pithon-Curi TC, Curi R. Neutrophil fatty acid composition: effect of a single session of exercise and glutamine supplementation. Amino Acids. 2008;35:243-5. doi:10.1007/s00726-007-0561-9.

27. Cadena PG, Pereira MA, Cordeiro RBS, Cavalcanti IMF, Barros Neto B, Pimentel MDCCB, et al. Nanoencapsulation of quercetin and resveratrol into elastic liposomes. Biochim Biophys Acta. 2013;1828:309-16. doi:10.1016/j.bbamem.2012.10.022.

28. Caddeo C, Teskac K, Sinico C, Kristl J. Effect of resveratrol incorporated in liposomes on proliferation and UV-B protection of cells. Int J Pharm. 2008;363:183-91. doi:10.1016/ j.ijpharm.2008.07.024.

29. Wieber A, Selzer T, Kreuter J. Physico-chemical characterisation of cationic DOTAP liposomes as drug delivery system for a hydrophilic decapeptide before and after freeze-drying. Eur J Pharm Biopharm. 2012;80:358-67. doi:10.1016/j.ejpb.2011.11.008.

30. Mendonça EAM, Lira MCB, Rabello MM, Cavalcanti IMF, Galdino SL, Pitta IR, et al. Enhanced antiproliferative activity of the new anticancer candidate LPSF/AC04 in cyclodextrin inclusion complexes encapsulated into liposomes. AAPS PharmSciTech. 2012;13:1355-66. doi:10.1208/s12249-012-9853-9.

31. Hamilton JG, Comai K. Separation of neutral lipids and free fatty acids by high-performance liquid chromatography using low wavelength ultraviolet detection. J Lipid Res. 1984;25:1142-8.

32. Koller M, Eckert H. Derivatization of peptides for their determination by chromatographic methods. Anal Chim Acta. 1997:352:31-59.

33. Snowden MK, Baxter JH, Bergana MM, Reyzer I, Pound V. Stability of n-acetylglutamine and glutamine in aqueous solution and in a liquid nutritional product by an improved HPLC method. J Food Sci. 2002;67:384-9.

34. Chorilli M, Salgado HRN, Santo FDS, Silva LM. Validation of a HPLC method for determination of glutamine in food additives using post-column derivatization. AJAC. 2012;3:113-7. doi:10.4236/ajac.2012.32016.

35. Lagranha CJ, Lima TM, Senna SM, Doi SQ, Curi R, Pithon-Curi TC. The effect of glutamine supplementation on the function of 
neutrophils from exercised rats. Cell Biochem Funct. 2005;23:101-7. doi:10.1002/cbf.1192.

36. Szebeni J, Muggia F, Gabizon A, Barenholz Y. Activation of complement by therapeutic liposomes and other lipid excipient- based therapeutic products: prediction and prevention. Adv Drug Deliv Rev. 2011;63:1020-30. doi:10.1016/j.addr.2011.06.017.

37. Newsholme P. Why is L-glutamine metabolism important to cells of the immune system in health, postinjury, surgery or infection? J Nutr. 2001;131:2515-22. 